\section{Comparative efficacy of inter- ferential therapy, bronchodila- tors, and body positioning on asthma control and quality of life of patients with bronchial asthma: A study protocol}

\author{
Eniola Awolola Oladejo, Sonill Maharaj \\ Sooknunan
}

Department of Physiotherapy, College of Health Sciences, University of KwaZuluNatal, Durban, South Africa

\begin{abstract}
Interferential Therapy (IFT) is the application of two medium frequency currents to the skin to stimulate and activate different systems in the body using specific frequencies and frequency ranges. The aim in applying IFT is to reduce myalgia in the chest and upper back, reduce muscular fatigue, and induce mucus expectoration. This study is designed to test the efficacy of IFT in reducing the symptoms frequently experienced by patients with bronchial asthma. Forty-eight (48) patients aged 18 and above with bronchial asthma attending the respiratory clinic at the Lagos State University Teaching Hospital, Ikeja (LASUTH) will be assessed for eligibility. The study design will be a double-blinded, randomized control trial with four intervention groups and four parallel placebo control groups. IFT will be administered as an intervention to patients on short and longacting bronchodilators in an assigned fundamental body position for 20 minutes. Six continuous outcome variables at different points will be utilized as outcome measures. A baseline Pulmonary Function Test (PFT) will be conducted on entry into the study while quality of life and asthma control will be evaluated every two (2) weeks during the study. Data obtained will be analyzed using descriptive and inferential statistics of repeated ANOVA; $\mathrm{P}<0.05$. The study outcome will compare the efficacy of IFT on bronchial asthma, identify its effect in different body positions, and compare the relationship between its application and the bronchodilator medication frequently used by the patients.
\end{abstract}

\section{Introduction}

Worldwide, approximately 300 million people are affected with bronchial asthma. ${ }^{1}$ It is more prevalent in developed countries, with the highest rates seen in Australia, UK, and New Zealand. ${ }^{1}$ In the Nigerian population, the prevalence of asthma ranges from $7 \%$ to $18 \%{ }^{1-4}$

A study by Oni et al., ${ }^{5}$ on the prevalence, management, and burden of asthma revealed that asthma affects all age groups, races, and sex. A population-based study showed that more boys are usually affected than girls in the early decades of life, however, the prevalence becomes equal by the third decade of life, and afterward. More women were reported to be affected after the third decade.

Symptom relief, reduction in the use of on-demand inhalers, improvement in activities and lung function are the day-to-day asthma control achievements. ${ }^{6}$ The absence of asthma exacerbations ensures the minimization of future risks by preventing accelerated decline in lung function and side-effects from medications over time. ${ }^{6}$

Progress is being made in the understanding and management of asthma, the inflammatory nature of the disease, use of steroids, and add-on of inhaled bronchodilator combined with steroids, devices to deliver the medications appropriately, and appreciation of the value of self-management education. ${ }^{1,7}$

In the treatment of airway disorders, bronchodilators are central in managing most chronic obstructive pulmonary diseases and are critical in managing asthma. ${ }^{8}$ The recent update by GINA ${ }^{9}$ indicates the consideration of low dose ICS-formoterol as controller medication for the management of bronchial asthma or ICS-SABA as an alternative in countries where ICS-formoterol is unavailable, low-dose.

According to Boros and MartusewiczBoros $^{10}$ airway reversibility is a test commonly used in diagnosing obstructive lung disease; its result can be used as a differential diagnosis between asthma and chronic obstructive pulmonary disease. Chung et $a l .{ }^{11}$ identified $\mathrm{FEV} 1 / \mathrm{FVC}<\mathrm{LLN}$ or 0.70 or $<L L N$ as a tool for diagnosing airway obstruction. Significant improvement (positive bronchodilatation test) is defined as an increase in FEV1 or FVC of more than 200 $\mathrm{mL}$, or $12 \%$ of the predicted value of FEV1. ${ }^{11}$ A bronchial provocative test can be used to determine the strength of the controller medication when FEV1 is $>70 \%$ predicted value. ${ }^{9}$

Several studies have been conducted to understand the effect of body position on pulmonary function. The most recent, a systematic review by Katz et al., ${ }^{12}$ reported higher FEV1, FVC, FRC, Imax, and PEF values in most studies involving healthy subjects or patients with lung, heart, neuromuscular disease, or obesity in a more erect
Correspondence: Eniola Awolola Oladejo, Department of Physiotherapy, College of Health Sciences, University of KwaZulu-Natal, Block E-5 University Road, Westville, Private Bag X54001, Durban, 4000, South Africa.

Tel.: +27679542461, +2348056333106

E-mail: 220068603@stu.ukzn.ac.za

Key words: IFT; asthma; PFT; ACT; AQLQ.

Acknowledgements: We hereby thank all the physiotherapists and medical doctors at Lagos State University Teaching Hospital, Ikeja, Lagos, for their contribution to the study's success and, most importantly, the participants for their voluntary participation. Our profound appreciation also goes to $\mathrm{Dr}$ Olufunke Adeyeye and Dr. Olufemi Ojo of Lagos State University Teaching Hospital, Ikeja, Lagos, for their professional advice in writing and reviewing this manuscript; to Miss Kemi and Miss Laide for their support in conducting the pulmonary function test; and to Miss Amodeni Ayomopewa for her editorial input.

Contributions: EAO developed the study idea; EAO and SMS developed the title; and both contributed to the study design. All authors were involved in designing the qualitative and quantitative aspects of the study. EAO was responsible for drafting the initial manuscript. SMS was responsible for editing and critical review of the manuscript. Both authors read, critically revised, and approved the final version of the manuscript. The views expressed in this study are strictly the views of the authors and not of the institution or any other group of people.

Conflict of interest: The authors declare no conflict of interest.

Funding: EAO funded the study. No funding was received from any external source for the study. The study design, writing the manuscript, data collection and analysis are independent of the institutions used for the study.

Access to Protocol: https://pactr.samrc.ac.za/ Researcher/ManageTrials.aspx The protocol was registered on the 1st of May 2020 with identifier number PACTR202005807526130 and the trial organization is UKZN

Availability of data and materials: The corresponding author will make available the datasets for the study upon reasonable request. However, the findings from the study will be made available to participating researchers as required by law.

Ethics approval and consent to participate: This study has been approved by the Biomedical Research Ethics Committee of the University of KwaZulu Natal (South Africa) (Ethics Number: BREC/00001883/2020), and by the Human Research Ethics Committee of Lagos State University Teaching Hospital, Ikeja, Lagos, Nigeria, West Africa (LREC/06/10/1428). The study is registered with ClinicalTrial.gov with registration number PACTR202005890624077.

Informed consent: A written and signed informed consent will be obtained from all participants recruited for this study through a third party that is independent of the study team. The consent form is designed by the Biomedical Research Ethics Committee of the University of KwaZulu-Natal (BREC) according to the WMA Helsinki Declaration and good clinical practice (GCP). During the trial, the PI will communicate in writing to the RECs in the event of the need to modify or amend the protocol, especially the inclusion or exclusion criteria of the study.

Received for publication: 24 October 2021

Revision received: 13 December 2021

Accepted for publication: 11 January 2022

This work is licensed under a Creative Commons Attribution 4.0 License (by-nc 4.0).

Copyright: the Author(s), 2022

Licensee PAGEPress, Italy

Healthcare in Low-resource Settings 2022; 10:10251

doi:10.4081/hls.2022.1051 
position. For subjects with tetraplegia, spinal cord injury, FVC and FEV1 were higher in supine vs. sitting. ${ }^{12}$

Interferential therapy (IFT) involves the application of two medium frequency currents to the skin in such a way that the currents "interfere" with each other to produce a "beat" frequency. ${ }^{13}$ The difference between the medium frequency currents is termed the beat frequency, and the body recognizes it as the required low-frequency current. Interferential therapy induces expectoration by making sputum on the bronchi surface mobile and reduces shoulder stiffness, muscular fatigue, and myalgia in the chest and upper back regions. ${ }^{14}$

Interferential current (IFC), a non-invasive treatment modality, is often used to induce analgesia, elicit muscle contractions, and reduce oedema. ${ }^{15-17}$ For many years, the effectiveness of asthma medications has been assessed by measuring their impact on expected clinical outcomes such as expiratory flow rates, symptoms, the need for other medications, and airway responsiveness. ${ }^{18}$ Although of great importance, none of the measurements indicates whether the patients can function better in their day-today lives. ${ }^{18}$

\section{Aim of the study}

The overall aim of this study is to determine the effect of IFT applied in $45^{\circ}$ or $90^{\circ}$ long sitting with a bronchodilator (short or long acting) on asthma control, quality of life, and selected pulmonary variables of asthmatic patients attending the respiratory clinic of Lagos State University Teaching Hospital, Ikeja, Lagos.

This study is designed to investigate the efficacy of a non-invasive therapeutic modality on airway reversibility, asthma control, quality of life of bronchial asthma (BA) patients in 2 different body positions using the GLI (LLN) 2012 reference equation for asthma classification and the 2019 Pan African Thoracic Society (PATS) and European Respiratory Society guidelines for the validation of spirometry test results. The outcome from this study may provide a non-invasive solution to the bronchospasm frequently experienced during an asthma attack.

\section{Hypothesis}

$\boldsymbol{H}_{0}$

Interferential therapy (IFT) applied in $45^{\circ}$ or $90^{\circ}$ long sitting will have no significant effect on airway reversibility, asthma control, and bronchial asthma patients' quality of life.

\section{Delimitation}

This study will be delimited to 48 bronchial asthma patients attending the Lagos State University Teaching Hospital, Ikeja, Lagos respiratory clinic.

\section{Significance of the study}

It is expected that the outcome of this study will establish the relationship among interferential therapy, bronchodilators, and body position in asthma control, asthma quality of life, and selected pulmonary variables of patients living with bronchial asthma. It is expected that this study will provide substantial evidence on airway reversibility in bronchial asthma using IFT.

\section{Materials and Methods}

\section{Study design}

The study is a parallel, 12-week randomized control trial and will involve four (4) intervention groups and four parallel placebo control groups.

\section{Participants}

The participants for this study will consist of male and female adult bronchial asthma patients aged 18 and above attending Lagos State University Teaching Hospital (LASUTH), Ikeja, Lagos, Nigeria in West Africa.

The inclusion criteria involve patients with bronchial asthma aged 18 and above attending the respiratory clinic of LASUTH.

The exclusion criteria involve patients with other types of COPD other than bronchial asthma, hypersensitive to B2 agonist, patients with a cardiac pacemaker, who have had recent surgery, supplemental oxygen therapy, or with cardiac conditions, and patients with psychological impairments.

Participants who meet the required criteria will be asked to read and sign an informed consent approved for this study by the appropriate institutional review board.

\section{Setting}

Patients with bronchial asthma attending the respiratory clinic of Lagos State University Teaching Hospital, Ikeja, Lagos State, Nigeria, will be recruited for the study. The hospital is a tertiary health facility within the state and receives referrals from within and outside the state.



Figure 1. F tests -MANOVA: Repeated measures, within-between interaction. 


\section{Sample size}

The pulmonary function test is the primary outcome of interest for the study and the expected clinically-relevant difference for pulmonary rehabilitation in various body positions using LLN and GLI reference equation proposed by Quanjer et al. ${ }^{19}$ Therefore, the sample size $(\mathrm{N})$ will be determined using the G-Power statistics software. The power is selected at $95 \%=0.95$, confidence level at $5 \%=0.05$ and effect size of 0.35 (Figure 1).

\section{Randomization and blinding}

The contact numbers of participants will be randomly extracted from the database on respiratory patients attending the Lagos State University Teaching Hospital Ikeja respiratory clinic (Figure 2). A bulk text message captioned "Invitation to a study on ASTHMA" will be circulated using the Luxury bulk SMS platform. Respondents will be assessed for eligibility, and those that meet the inclusion criteria will participate in the study while those who have any of the exclusion criteria will be excluded.

Participants will be randomly selected by simple randomization using a computer software program randomization table. ${ }^{20}$ The software program (www.randomization.com) will be used to allocate participants into study group A and control group B. Group A was further assigned to subgroup $\mathrm{x}$, and $\mathrm{y}$, while group B was assigned to subgroup e, and f. Subgroup $x$ and e represents participants on SABA, while Subgroup y and $\mathrm{f}$ represents participants on LABA. Participants were randomly assigned to a final group of $x a, x b, y a, y b$, ea, eb, fa, and $\mathrm{fb}$, with ' $\mathrm{a}$ ' and ' $\mathrm{b}$ ' connoting $45^{\circ}$ and $90^{\circ}$ long sitting respectively.

\section{Procedure for data collection}

Forty-eight (48) subjects will be recruited for this study. The subjects will be randomly assigned into two major groups of twenty-four (24) subjects per group, two (2) subgroups of twelve (12) subjects per subgroup, and eight (8) final groups of six (6) subjects per group.

\section{Assessment}

The subjects' medical records will be adequately screened for possible contraindications to the study. Baseline respiratory parameters will be assessed using the Koko PFT spirometer.

The Asthma Control Test Questionnaire and Asthma Quality of Life questionnaire will be administered to the control and study groups at the beginning of the study and at two-week intervals for the 12 weeks of the study. Baseline spirometry will be

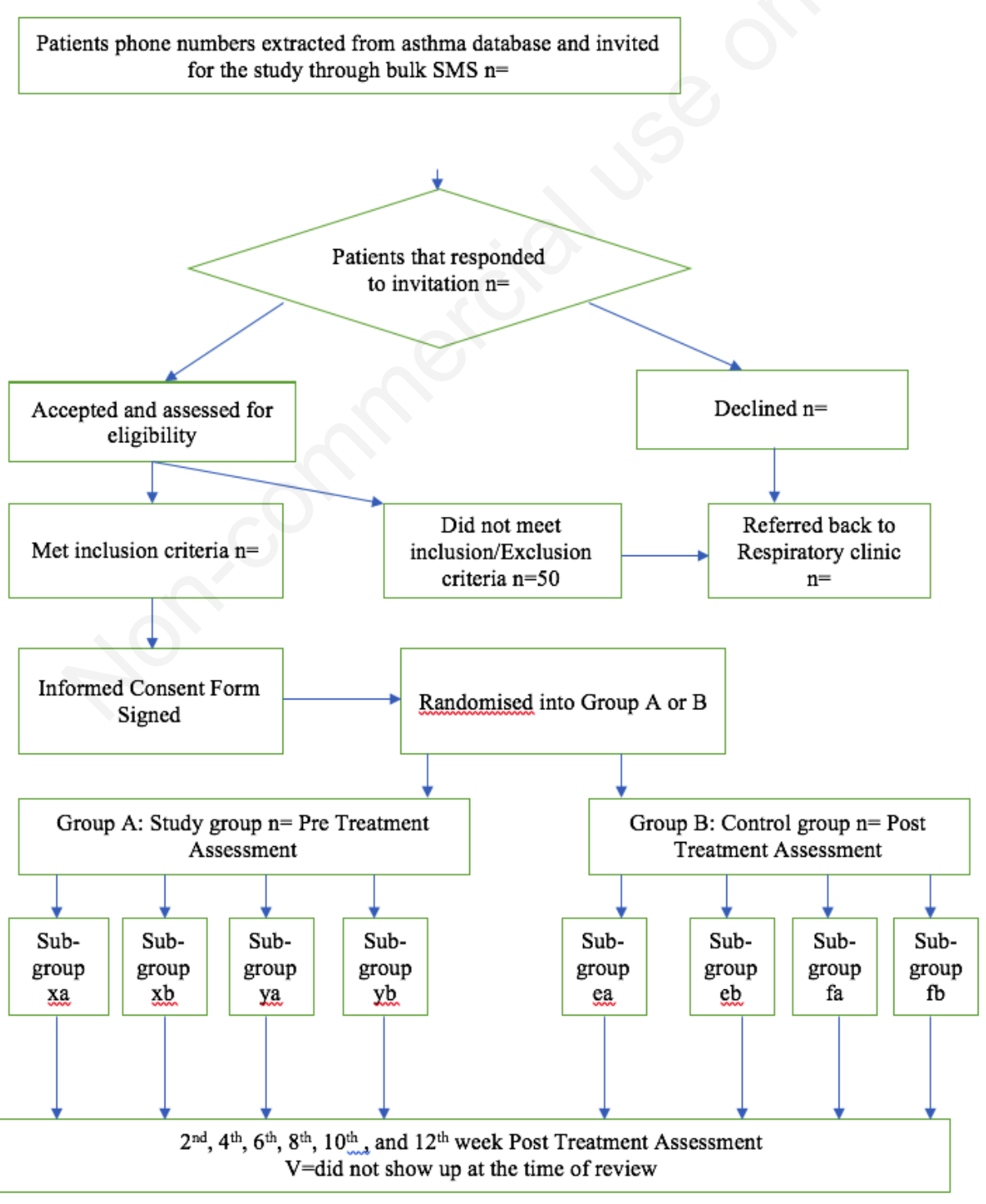

Figure 2. Recruitment and randomization of participants. 
conducted on the two groups before and after the commencement of the study.

The interferential unit "Nu-Tek E-Stim Pro MT1022970" will be used for the study intervention. The treatment period will be increased by two minutes with each application, up to a total application time of 20 minutes.

\section{Assessment tools}

\section{Spirometry assessment}

The assessment will be conducted by a spirometrist certified by the Pan African Thoracic Society. A portable spirometer (Koko SX 1000 Standalone Version 7 Pneumotach) will be used to carry out this assessment. Daily calibration of the device will be done using a 3.0-litre syringe. A brief description of the assessment procedure, including technical steps to obtain pulmonary function data and variables, will be explained to each subject. After 2-3 tidal breaths, the subject will be asked to inhale deeply to total lung capacity and then exhale rapidly (without any pause) through a disposable mouthpiece until as much air as possible has been expelled from the lungs. The test will be performed in a sitting or standing position. The assessments will be repeated three times after adequate rest. The maximum number of attempts permitted will be 8 . After fulfilling the acceptability and repeatability criteria, the two best curves will be selected. The average values of the forced vital capacity (FVC) and forced expiratory volume in the first second (FEV1) will be recorded. ${ }^{21}$

\section{Intervention}

Participants will be briefed about the nature, effect, and benefit of the study. They will be encouraged to clarify issues regarding the study. All participants will be required to give a written informed consent before participating in the study. Participants will be randomly assigned into two groups: study group (Group A) and control group (Group B). Interferential therapy will be demonstrated to the study group alone. The Asthma Control Test, Asthma Quality of Life Questionnaire, and spirometry score will be measured and recorded before intervention in both groups. Reassessments will conducted at the end of the $2 \mathrm{nd}, 4 \mathrm{th}, 6 \mathrm{th}, 8 \mathrm{th}, 10 \mathrm{th}$, and 12 th weeks of the study intervention.

The study group (Group A) will receive interferential therapy; this modality selection is only acceptable in the absence of cardiac disease history. ${ }^{22}$ In the absence of such a history, the subjects will be divided into two subgroups and labeled $\mathrm{x}$ and $\mathrm{y}$. Subgroup ' $\mathrm{x}$ ' will be allowed to use 400ug of SABA delivered in metered doses via a spacer4-6 hours before the study, while subgroup ' $y$ ' will be allowed to use $24 \mathrm{ug}$ of LABA delivered in metered doses via a drypowder inhaler within 24 hours of the study. ${ }^{23}$ In both cases, the inhaler technique, as described by Basheti et al. ${ }^{24}$ will be used to demonstrate the procedure to the participants. The intervention will be conducted with participants in a long sitting position, at an angle of $45^{\circ}$ and $90^{\circ}$ representing labels ' $a$ ' and ' $b$ ' respectively. In both intervention positions, an electrode will be placed bilaterally over the upper limit of the trapezius on the upper back and the other two anteriorly over the lower ribs. ${ }^{22}$ The final group of participants to undergo the intervention will consist of ' $x a$,' 'xb,' ' $y a$ ' and 'yb.' If the subject experiences respiratory difficulty during the intervention, the procedure will be discontinued. With a $4,000 \mathrm{~Hz}$ base current, the interferential current range will be set between 10 to $150 \mathrm{~Hz}$ and initially applied for 10 minutes, while carefully monitoring the patient's condition during the treatment period. If the patient shows any sign of distress during the treatment, the current will be turned off. As long as the subject does not experience any distress with the IFC application, the treatment period will be increased by two minutes with each application, for up to 20 minutes.

Participants in the control group (Group B), in addition to the baseline pulmonary function test, will also receive free musculoskeletal assessment and counselling on asthma. They will be divided into two subgroups and labelled 'e' and 'f.' Participants in subgroup 'e' will be allowed to use 400ug of SABA delivered in metered doses via a spacer 4-6 hours before the study, while subgroup ' $f$ ' will be allowed to use 24ug of LABA delivered in metered doses via a drypowder inhaler within 24 hours of the study. ${ }^{23}$ The intervention will be conducted with participants in a long sitting position, at an angle of $45^{\circ}$ and $90^{\circ}$ representing labels ' $a$ ' and ' $b$ ' respectively. The final group of participants to undergo the intervention will consist of 'ea,' 'eb,' 'fa' and ' $f b$.' In both cases, the inhaler technique, as described by Basheti et al, ${ }^{24}$ will be used to demonstrate the procedure to the participants. They will be asked to maintain their respective positions for 20 minutes. If the subject experiences respiratory difficulty, the procedure will be discontinued.

\section{Outcome measures/instruments}

i) Asthma Control Test (ACT); ii) Asthma Quality of Life Questionnaire (AQLQ); iii) Spirometer (Koko SX 1000 Standalone Version 7 Pneumotach); iv) Interferential Therapy Machine (Nu-Tek EStim Pro MT1022970).

\section{Description of outcome measures/instruments}

\section{Asthma Control Test (ACT)}

The asthma control test is a self-administered 5-item questionnaire developed for assessing asthma control level. It evaluates the most recent four-week period. Each item is scored between 1 and 5, with a total score ranging from 5 to 25 . An ACT score of 25 indicates that asthma is "controlled" whereas a score between 20 and 24 shows partially controlled asthma and a score of $<20$ indicates "uncontrolled" asthma. ${ }^{25}$

\section{Asthma Quality of Life Questionnaire Standardised (AQLQS)}

The standardized version of the Asthma Quality of Life Questionnaire (AQLQ) is a 32-item questionnaire (self-administered or clinician administered) with five domains, developed to measure the functional, physical, emotional, occupational, and social problems that are most troublesome to adults with asthma. ${ }^{18}$ The maximum score obtainable is 7.0, which translates to no impairment. The minimum score is 1.0 , indicating severe impairment, 4.0 is the mid-range score, and indicates moderate impairment. ${ }^{18}$ The Asthma Quality of Life Questionnaire standardized version will be used to monitor the difficulty the subjects encounter in activities of daily living as a result of asthma.

\section{Spirometer}

Koko SX 1000 Standalone Version 7 Pneumotach, a portable lightweight and comprehensive diagnostic tool, will be utilized to conduct the pulmonary function test. The Koko Legend II spirometer has a built-in thermal printer and a touch screen display. It can perform FVC, Pre vs. Post, and SVC tests. Test data and patient information are stored directly on an internal SD card that can be replaced and re-used. All the stored information can be downloaded via a USB cable onto a PC for backup or storage. This device supports daily calibration checks, complies with ATS-ERS 2005, has several predicted authors, and includes GLI-2012. Daily calibration of the device will be conducted using a $3 \mathrm{~L}$ syringe. ${ }^{26}$

The participant's condition can be shown by the ratio of the measured value to the predicted value. Flow rate-volume chart, volume-time chart display, data memory, delete, upload and review, trend chart display, scaling (calibration), information prompts when volume or flow goes beyond the limits are features available on the device.

\section{Bronchodilators}

The administration of bronchodilators will be primarily through inhalation devices 
to deliver the drug to the lung bronchioles in metered doses. Inhalation devices come in all shapes and sizes, but critical is maximizing the amount of drug reaching the bronchioles. The best way to achieve maximum bioavailability is by fully exhaling, placing the inhaler in the mouth, and taking a full inhalation. After the patient has inhaled completely, it will be followed by $10 \mathrm{sec}$ onds of no breathing to wait for the medicine to dissipate into the lung space. A slow exhalation back to normal breathing will be advised. ${ }^{27,28}$ The eight-point inhaler technique as described by Basheti, Natsheh $^{24}$ will be used to deliver bronchodilators and pre-BD reversibility testing in metered doses.

\section{Interferential unit}

The Nu-Tek Electrotherapy Machine EStim Pro MT1022 provides low and medium frequency outputs from a single unit. The currents available on the $\mathrm{Nu}$-Tek Electrotherapy Machine E-Stim Pro MT1022 include Interferential (2 and 4pole), Russian, Diadynamic, TENS, Sinusoidal, Faradic, Galvanic, Interrupted Galvanic, Trabert, and Medi-Wave. ${ }^{29}$ The Nu-Tek E-Stim Pro MT1022 will be used to deliver interferential current by generating a beat frequency range of $10-150 \mathrm{hz}$ from two medium frequency currents undulating at a base frequency of $4000 \mathrm{~Hz}$ to $4100 \mathrm{~Hz} .{ }^{14} \mathrm{An}$ interferential current will be activated with two electrodes, one placed posteriorly at the upper border of the trapezius and the other anteriorly below the ribs. A beat frequency will be generated at the point of intersection, resulting in relaxation of the smooth muscles, resolution of pain, and mobilization of secretions. ${ }^{14}$

\section{Data analysis}

The Statistical Package for Social Sciences (SPSS Inc, Chicago, II) version 26.0 for the Windows package program will be used to analyse data. The results will be summarized using descriptive statistics of mean, standard deviation, frequency, and percentages. Bar charts, pie charts, and histograms will be utilized for pictorial illustration. A multilevel analysis of variance (ANOVA) will be used to compare the outcome variables [body position (45 degrees long sitting and 90 degrees long sitting), pulmonary function variables (FEV1, FVC, FEV1/FVC), asthma control test (ACT), and the standardized Asthma Quality of Life Questionnaire (AQLQ)] among each group, and the dependent t-test will be used to compare the pre and post-test results while the independent t-test will be used to compare the outcome variables across the two groups. The level of significance will be set at $\mathrm{p} 0.05$.

\section{Harms}

This study carries minimal risks. The procedures are not life-threatening and should not cause any harm or negative effect. The effects may include temporary muscle soreness, increased heart rate, blood pressure, sweating, and dizziness. Necessary care will be taken to prevent the occurrence of an adverse event. However, in case of a report of serious adverse events (e.g., comorbidities, injuries, persistent excruciating pain, dizzy spells, headache, etc.) after intervention or at any point during the trial, we would consider unblinding the participant to the intervention for his/her safety. Additionally, the participants will be instructed to report any adverse events to the PI or the physiotherapist supervising their group. To ensure adequate supervision and safety, the number of participants per group in a day will be limited to a maximum of 3. Arrangements have been made with the Accident and Emergency unit of the hospital where the research will be conducted to provide a standby medical team. However, the University of KwaZulu-Natal insurance scheme on clinical trials fully covers participants in this type of study.

\section{Discussion}

The relationship between medicationinduced airway reversibility and reversibility obtained through electrophysical modalities is still not well justified. Furthermore, the relationship between the mode of delivery of electrophysical agents and the recovery pattern in bronchial asthma is yet to be fully understood.

A study by Karashurov et al..$^{30}$ on programmed electrostimulation of the sinocarotid nerves implanted to 78 patients with bacterial asthma for six years was reported to have prevented the majority of asphyxia attacks, reduced their frequency 2.7-fold, and the need for medications 2.73.4-fold.

Aweto et al. ${ }^{31}$ in a study of the effect of IFT on the cardiopulmonary parameters of 42 BA patients for six weeks reported a significant improvement in systolic blood pressure $(\mathrm{p}=0.004)$, forced expiratory volume in one second $(\mathrm{p}=0.02)$, forced vital capacity $(\mathrm{p}=0.04)$, and peak expiratory flow rate $(\mathrm{p}=0.007)$, while the control group had significant reductions in pulmonary parameters. There were significant improvements (increases) in the ACT score $(p=0.0001)$ and AQLQ ( $\mathrm{p}=0.001)$.

Mohammed and Elyazed ${ }^{32}$ studied thirty Egyptian children aged 9-15 with BMI 18.5 to $24.9 \mathrm{~kg} / \mathrm{m}^{2}$, who had asthma. The pre- and post-treatment variables revealed a significant improvement in pulmonary functions in favor of laser puncture therapy and interferential therapy over diaphragmatic exercise.

Although studies by Aweto et al., ${ }^{31}$ Karashurov et al., ${ }^{30}$ and Mohammed and Elyazed $^{32}$ identified the effect of an electrophysical agent in the management of asthma, their findings did not ascertain the possible effect of the medication used by the patients during the procedure. Consequently, it is expected that this study's outcome will further reveal the effect of the electrophysical modality on the symptoms frequently experienced by asthma patients who are on short or long-acting bronchodilator medication.

Finally, it is expected that the findings of this study could serve as guideline for the management of BA with electrophysical agents and would further support the costbenefit of asthma management in Nigeria and other low-income countries.

\section{References}

1. Masoli M, Fabian D, Holt S, Beasley R. Global Initiative for Asthma (GINA) Program. The global burden of asthma: Executive summary of the GINA Dissemination Committee report. Allergy 2004:59:469-478.

2. Desalu OO, Oluboyo OP, Salami AK. The prevalence of bronchial asthma among adults in Ilorin, Nigeria. Afr J Med Med Sci 2009:38:149-54.

3. Ibe CC, Ele UP. Prevalence of bronchial asthma among adolescents in Anambra State, Nigeria. Nigeria J Int Med 2002:5:23-6.

4. Erhabor GE, Agbroko S, Bamigboye P, Awopeju OF. Prevalence of asthma symptoms among university students 15 to 35 years of age in Obafemi Awolowo University, Ile-Ife, Osun State. J Asthma 2006:43:161-4.

5. Oni AO, Erhabor GE, Egbagbe EE. The prevalence, management and burden of asthma - a Nigerian study. Iran J Allergy Asthma Immunol 2010;9:35-41.

6. National Asthma Education and Prevention Program, Third Expert Panel on the Diagnosis and Management of Asthma. Expert Panel Report 3: Guidelines for the Diagnosis and Management of Asthma. Bethesda (MD): National Heart, Lung, and Blood Institute (US); 2007 Aug. Available from: https://www.ncbi.nlm .nih.gov/books/NBK7232/

7. Oni AO, Erhabor GE, Egbagbe EE. The prevalence, management and burden of asthma-A Nigerian study. Iran J Allergy 
Asthma Immunol 2010:1:35-41.

8. Cazzola M, Page CP, Calzetta L, Matera MG. Pharmacology and therapeutics of bronchodilators. Pharmacology Rev 2012:3:450-504.

9. GINA. Global Initiative for Asthma. Global strategy for asthma management and prevention, 2021. Available from: www.ginasthma.org

10. Boros PW and Martusewicz-Boros MM. Reversibility of airway obstruction vs bronchodilatation: do we speak the same language? COPD 2012;9:213215.

11. Chung KF, Wenzel SE, Brozek JL. International ERS/ATS guidelines on definition, evaluation and treatment of severe asthma. Eur Respir J 2014;43:343-73.

12. Katz S, Arish N, Rokach A. The effect of body position on pulmonary function: a systematic review. BMC Pulm Med 2018;18:159.

13. Emberson W. Asthma interferential therapy and chartered physiotherapy. Positive Helath Online 1999;36.

14. Shuto H, Nakagami K, Suzuki H, Noguchi E. Low-frequency interference in bronchial asthma. Jap J Allergol 1986;35:12:1170-80.

15. Goats GC. Interferential current therapy. Br J Sports Med 1990:24:87-92.

16. McManus FJ, Ward AR, Robertson VJ. The analgesic effects of interferential therapy on two experimental pain models: cold and mechanically induced pain. Physiotherapy 2006:92:95-102.
17. Ozcan J, Ward AR, Robertson VJ. A comparison of true and premodulated interferential currents. Arch Phys Med Rehabil 2004;85:409-415.

18. Juniper EF, Guyatt GH, Ferrie PJ. Measuring quality of life in asthma. Am Rev Respir Dis 1993;147:832-8.

19. Quanjer PH, Enright PL, Miller MR. Open letter: the need to change the method for defi ning mild airway obstruction. Prim Care Respir J 2010:19:288-91.

20. Suresh K. An overview of randomization techniques: An unbiased assessment of outcome in clinical research. J Hum Reprod Sci 2011;4:8-11.

21. Masekela R, Zurba L, Gray D. Dealing with access to spirometry in Africa: a commentary on challenges and solutions. Int J Environ Res Public Health 2019;16:62.

22. Davis V. Management of asthma. Dynamic Chiropractic J 1992;10.

23. Wallin A, Sandstrom T, Rosenhall L, et al. Time course and duration of bronchodilatation with formoterol dry powder in patients with stable asthma. Thorax 1993;48:611-4.

24. Basheti IA, Natsheh AI, Ammari WG. Education on correct inhaler technique in pharmacy schools: Barriers and needs. Tropical J Pharm Res 2015;14:715-22.

25. Nathan RA, Sorkness CA, Kosinski M. Development of the asthma control test: a survey for assessing asthma control. J Allergy Clin Immunol 2004;113:59-65.
26. nSpire. KoKoPfT. 2020. Accessed 04/02/21. Available from: https://www.kokopft.com/

27. Feng JF, Ding GR, Xie YZ. Efficacy of budesonide/formoterol and tiotropium combination for the treatment of Chinese patients with chronic obstructive pulmonary disease. Medicine (Baltimore) 2018; 97: e10841.

28. Hanania NA, Sethi S, Koltun A. Longterm safety and efficacy of formoterol fumarate inhalation solution in patients with moderate-to-severe COPD. Int $\mathrm{J}$ Chron Obstruct Pulmon Dis 2019;14:117-27.

29. Nu-Tek. Nu-Tek Electrotherapy Machine E-Stim Pro MT1022. Accessed 4/02/21. http://www.nutekmedical.com/product/news94.html

30. Karashurov SE, Gudovskii LM, Semenova LI. [Electrostimulation in the therapy of bronchial asthma]. Klin Med (Mosk) 2001;79:39-41.

31. Aweto HA, Tella B. Awolola EO. Efficacy of interferential therapy on selected cardiopulmonary parameters, asthma control and quality of life of people living with asthma. Romanian J Physical Ther 2016;21.

32. Mohammed AH and Elyazed TIA. Laser puncture therapy versus interferential therapy as a combined treatment in asthmatic egyptian children: Comparison of treatment approaches. Int J Physiother Res 2017;5:2457-63. 